\title{
LA SUFICIENCIA TAXONÓMICA COMO HERRAMIENTA PARA EL MONITOREO DE ARTRÓPODOS EPÍGEOS: UNA PRIMERA APROXIMACIÓN EN EL DESIERTO COSTERO PERUANO
}

\author{
TAXONOMIC SUFFICIENCY AS A TOOL FOR MONITORING EPIGAEIC \\ ARTHROPODS: A FIRST ATTEMPT IN THE PERUVIAN COASTAL DESERT
}

\author{
Alfredo Edgardo Giraldo Mendoza ${ }^{1}$
}

\begin{abstract}
Resumen
La suficiencia taxonómica ha recibido creciente atención en estudios sobre el monitoreo de los efectos de perturbaciones en comunidades de artrópodos terrestres. El objetivo principal fue probar que la estructura comunitaria de artrópodos epígeos analizada a distintas resoluciones taxonómicas puede distinguir los ecosistemas en estudio. Los artrópodos epígeos fueron colectados con trampas de caída en seis ecosistemas del desierto costero peruano, en la provincia de Pisco (Ica, Perú). La capacidad de distinguir los ecosistemas fue comparada entre órdenes, familias y especies, utilizando tres tipos de análisis multivariados (NMDS, cluster, ANOSIM) y tres índices de similitud (Raup-Crick, Bray-Curtis, Morisita-Horn). Asimismo, se realizaron análisis adicionales para caracterizar las matrices de datos y, medir la relación de la estructura comunitaria con el principal gradiente ambiental (aridez). Los resultados fueron: 1) la estructura comunitaria de artrópodos epígeos analizada a distintas resoluciones taxonómicas distinguió los ecosistemas incluidos en el área de estudio; 2) la agregación de las matrices de datos es un proceso que mejora la aplicación de los análisis multivariados; 3) la estructura comunitaria de artrópodos epígeos analizada a distintas resoluciones taxonómicas estuvo relacionada al principal gradiente ambiental del área de estudio. Estos resultados fueron comparados con los hallazgos de estudios previos y se formulan algunas sugerencias para estudios futuros.

Palabras clave: artrópodos epígeos, estructura comunitaria, suficiencia taxonómica, índices de similitud, monitoreos biológicos, datos multivariados.
\end{abstract}

\begin{abstract}
Taxonomic sufficiency has received increasing attention in studies aimed at monitoring the effects of disturbances on terrestrial arthropod communities. The main objective was to prove that the community structure of epigaeic arthropods analyzed at different taxonomic resolutions can distinguish the ecosystems studied. Epigaeic arthropods were sampled with pitfall traps in six Peruvian coastal desert ecosystems in the province of Pisco (Ica, Peru). The ability to distinguish ecosystems was compared among orders, families and species, using three types of multivariate analyzes (NMDS, cluster, ANOSIM) and three similarity indexes (Raup-Crick, Bray-Curtis, Morisita-Horn). Also, additional analyses were performed to characterize the data matrices and to measure the relationship of community structure with the main environmental gradient (aridity). The results were: 1) the community structure of epigaeic arthropods analyzed at different taxonomic resolutions distinguished ecosystems included in the study area, 2) the aggregation of data matrices is a process that improves the application of multivariate analysis, 3) the community structure of epigaeic arthropods analyzed at different taxonomic resolutions was related to the main environmental gradient in the study area. These results are compared with findings from previous studies and some suggestions for future studies are made.

Key words: epigaeic arthropods, community structure, taxonomic sufficiency, similarity indexes, biological monitoring, multivariate data.
\end{abstract}

Introducción.

Llevar a cabo monitoreos biológicos en periodos relativamente cortos es una necesidad actual en el contexto de la creciente y acelerada modificación humana de los ecosistemas terrestres (Vitousek et al., 1997). Sin embargo, el inventario de los organismos vivientes más diversos es aún un proceso incompleto que requiere un largo plazo para ser concluido (May, 
1990). En el caso de los artrópodos terrestres, asignar los ejemplares colectados en localidades concretas a sus respectivas especies no siempre es una tarea simple y directa, especialmente en los países ubicados en los trópicos. Esta problemática ha sido llamada impedimento taxonómico, la dificultad para reunir los recursos humanos y materiales necesarios para que las labores de determinación taxonómica sean cumplidas con éxito (Cardoso et al., 2011). Frente a este panorama se han propuesto una serie de opciones metodológicas, las cuales han sido recomendadas para evaluar la fauna de artrópodos terrestres en varios países, incluyendo Australia (New, 1996), Sudáfrica (Slotow \& Hamer, 2000) y Nueva Zelanda (Ward \& Larivière, 2004).

La suficiencia taxonómica o agregación taxonómica es una de tales opciones metodológicas. Es el procedimiento por el cual los especímenes son clasificados hasta un nivel de resolución taxonómica que sea suficiente para satisfacer los objetivos de un estudio (Ellis, 1985). Este es un concepto pragmático, que busca evitar la labor y el costo asociados a la determinación taxonómica de especies, asumiendo que los cambios multivariados en la estructura de comunidades biológicas pueden ser evidentes tanto a nivel de especies como a nivel de taxones de alto rango (Pik et al., 1999). La suficiencia taxonómica ha recibido considerable atención en investigaciones acerca de los efectos de la polución antropogénica sobre las comunidades de invertebrados bentónicos en aguas continentales (Jones, 2008) y marinas (Warwick \& Clarke, 1993; Terlizzi et al., 2003). Las investigaciones realizadas en este campo coinciden en señalar que la polución puede diagnosticarse con una precisión equivalente a partir de datos a nivel de especies, géneros, familias, órdenes o incluso phyla.

Las aplicaciones iniciales de la suficiencia taxonómica en artrópodos terrestres estuvieron referidas a los efectos de la reforestación (Pik et al., 1999) y la fragmentación (Major et al., 1999) sobre la fauna epígea de Coleoptera y Formicidae en bosques de Australia. Desde entonces, se han sumado investigaciones que abarcan distintos grupos de artrópodos terrestres, ecosistemas y perturbaciones antrópicas (Pik et al., 2002; Caruso \& Migliorini, 2006; Biaggini et al., 2007; Uehara-Prado et al., 2009; Groc et al., 2010; Bang \& Faeth, 2011). Un reciente meta-análisis de estudios de biodiversidad de artrópodos en Canadá (Timms et al., 2013) sugiere que la suficiencia taxonómica debería aplicarse a la resolución taxonómica de género y cuando la proporción taxonómica sea baja, es decir si las familias o los géneros incluyen pocas especies.

El principal objetivo del presente trabajo fue demostrar que la suficiencia taxonómica aplicada a datos de artrópodos epígeos no provoca una significativa pérdida de información multivariada. Esta hipótesis fue probada en comunidades de artrópodos epígeos, cuya estructura comunitaria fue utilizada para distinguir una serie de ecosistemas en el desierto costero peruano. Los objetivos secundarios fueron: comprobar que agregar las matrices de datos mejora la aplicación de los análisis multivariados y, que la estructura comunitaria de artrópodos epígeos a distintas resoluciones taxonómicas puede relacionarse al gradiente ambiental reconocido en el área de estudio.

\section{Materiales y métodos.}

Área de estudio.

El estudio fue realizado en la región Ica, provincia de Pisco, distritos de San Andrés y Paracas. Se establecieron nueve puntos de muestreo repartidos equitativamente en tres localidades que incluyen distintos ecosistemas del desierto costero peruano:

La Planta de Fraccionamiento de Líquidos de Gas Natural (Pluspetrol Perú Corporation S.A.)

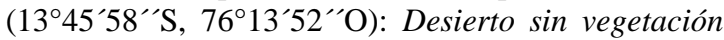
en los puntos de muestreo PF1 y PF2, áreas totalmente desprovistas de vegetación, completamente expuestos a la radiación solar, suelo arenoso-pedregoso, sin fuentes de agua, sin instalaciones pero expuestas a tránsito peatonal y vehicular. Vegetación introducida en el punto de muestreo PF3, área intervenida por un proyecto de mejoramiento paisajístico, con vegetación arbórea sembrada que incluye entre otras a Acacia horrida, Eucalyptus robusta, Myoporum laetum, Prosopis pallida y Vachellia macracantha, combina áreas sombreadas con otras expuestas a la radiación solar, suelo arenoso-pedregoso con parches de mantillo, agua suministrada mediante riego.

El bosquecito de Santa Cruz (Reserva Nacional de

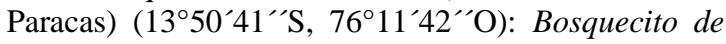
sofaique en los puntos de muestreo SC1 y SC2, vegetación dominada por la especie vegetal de porte arbóreo Geoffroea decorticans, áreas sombreadas, suelo arenoso cubierto con mantillo, agua de napa freática, en áreas previamente utilizadas para la extracción de madera. Zona de palmeras en el punto de muestreo SC3, vegetación mixta de palmera datilera (Phoenix dactylifera) y herbáceas (dominadas por Distichlis spicata), combina áreas sombreadas con otras expuestas a la radiación solar, suelo cubierto con costras calcáreas interrumpidas por áreas arenosas, sin fuentes de agua, en un área previamente utilizada para la agricultura.

El humedal de San Andrés (1343'12"s, 76¹3'19"O): Humedal poco perturbado en los puntos de muestreo SA1 y SA2, vegetación herbácea dominada por Paspalum vaginatum, Salicornia fruticosa y Distichlis spicata, parcialmente expuestos a la radiación solar, suelo arenoso húmedo, agua de napa freática, área aledaña a cuerpos de agua con totorales. Humedal muy perturbado en el punto de muestreo SA3, vegetación herbácea dominada por Paspalum vaginatum, Salicornia fruticosa y Distichlis 
spicata, parcialmente expuesto a la radiación solar, suelo arenoso compactado, agua de napa freática, área expuesta a una mayor presión antrópica (infiltración de desagües, residuos sólidos).

En síntesis, los nueve puntos de muestreo pueden ser clasificados en seis ecosistemas a partir de sus características de suelo, vegetación y perturbación antrópica. Asimismo, se reconoce la existencia de un gradiente ambiental de aridez, en el cual los puntos de muestreo PF1, PF2 y SC3 se ubicaron en el extremo de mayor aridez y los puntos de muestreo SA1, SA2, SA3 y PF3 en el extremo de menor aridez.

Métodos de campo y laboratorio.

Los artrópodos epígeos fueron colectados con trampas de caída (Ausden, 1996; Márquez-Luna, 2005). Las trampas fueron vasos de plástico de $7.5 \mathrm{~cm}$ de diámetro, enterrados al ras del suelo y llenos a la mitad de su capacidad con una solución conservante compuesta por agua (70\%), refrigerante (29\%) y detergente (1\%). En cada punto de muestreo se colocaron 10 trampas de caída, con una distancia de cinco metros entre sí, las cuales permanecieron en campo durante 48 horas. Este procedimiento fue repetido con frecuencia semestral hasta completar seis evaluaciones (Abril 2011, Marzo 2012 y 2013, Septiembre 2011, 2012 y 2013). Las muestras fueron conservadas en frascos de plástico con etanol 70\%, debidamente etiquetadas y trasladadas al laboratorio para su revisión.

En el laboratorio, las muestras se examinaron en placas petri bajo un microscopio estereoscópico (aumento entre 20-40x), para separar los artrópodos de los residuos de sustrato y material vegetal, realizar su determinación taxonómica y contar el número de ejemplares colectados.

Todos los ejemplares fueron determinados a nivel de órdenes empleando las claves de Arnett (1985) y Barrientos (1988), considerando los estadíos larvales de los órdenes insectos como grupos separados. Los ejemplares de los órdenes Araneae, Coleoptera, Diptera, Hemiptera e Hymenoptera fueron determinados a nivel de familias (o superfamilias) empleando las claves de Aguilera \& Casanueva (2005), CSIRO (1991) y McAlpine et al. (1981). Estos cinco órdenes abarcaron la mayor parte de la diversidad taxonómica (familias y especies) y funcional (detritívoros, fitófagos, predadores, parasitoides, etc.) de los artrópodos terrestres. Los ejemplares de las familias Gnaphosidae, Lycosidae, Oonopidae, Oxyopidae y Salticidae del orden Araneae; Carabidae, Histeridae, Anobiidae (Ptininae), Staphylinidae y Tenebrionidae del orden Coleoptera; y Formicidae del orden Hymenoptera fueron determinados a nivel de especies. La determinación de especies tuvo un resultado heterogéneo, especies reconocidas, especies en géneros reconocidos (especies no descritas, géneros en revisión) y morfoespecies, es decir conjuntos de individuos que se consideran especies diferentes a partir de su morfología externa (Oliver \& Beattie, 1996). En algunos casos, se contó con claves para la determinación de géneros neotropicales (Mazur, 2001; Navarrete-Heredia et al., 2002; Palacio \& Fernández, 2003; Martínez, 2005). Las 11 familias seleccionadas presentaron al menos alguno de los siguientes atributos: fueron eficientemente colectadas por las trampas, estuvieron presentes en varios de los puntos evaluados y/o un adecuado conocimiento taxonómico. La determinación de los órdenes, la mayoría de familias y las especies de Coleoptera y Formicidae fue realizada por el autor. La determinación de las familias y especies de Araneae fueron realizadas por MDB (ver agradecimientos). Los ejemplares han sido incorporados a la colección del Museo de Entomología "Klaus Raven Buller" - Universidad Nacional Agraria La Molina (Lima, Perú).

Análisis de datos.

Los datos obtenidos en las seis evaluaciones fueron acumulados y organizados en matrices de taxones $\mathrm{x}$ puntos de muestreo en los tres niveles de resolución taxonómica antes señalados: órdenes, familias y especies.

Para distinguir los ecosistemas evaluados a partir de la estructura comunitaria de artrópodos epígeos se realizaron tres tipos de análisis multivariados: Un escalamiento no métrico multidimensional (NMDS), un análisis de conglomerados (cluster) y un análisis de similitudes (ANOSIM) (Legendre \& Legendre, 1998). El NMDS es un método de ordenación utilizado para colocar los puntos de muestreo en un sistema de coordenadas bidimensional de tal manera que sus diferencias en similitud sean preservadas. En este caso, el programa estadístico hizo una selección automática de la solución con menor stress de un total de 11 ensayos. El análisis de conglomerados sirvió para generar grupos de puntos de muestreo a partir de las relaciones de similitud existentes entre las comunidades. Se utilizó un método jerárquico, el método de agrupamiento por medias aritméticas (UPGMA), y el valor de 0.66 como referencia para reconocer los grupos en una escala de similitud de 0 a 1. El ANOSIM es una prueba no paramétrica que sirvió para determinar la significación estadística de los grupos obtenidos mediante el análisis de conglomerados. El programa estadístico calculó el estadístico R, el cual denota disimilitud entre grupos si su valor se aproxima o sobrepasa a 1. La significación estadística de dicho coeficiente se obtuvo mediante un proceso de aleatorización, y se optó por un valor referencial de $p<0.05$. Antes de realizar los análisis multivariados, los datos de las matrices fueron sometidos a una transformación logarítmica $\left(\log _{10}(n+1)\right)$, para reducir la influencia de los taxones dominantes (Legendre \& Gallagher, 2001).

Los análisis descritos fueron replicados para tres índices de similitud: Raup-Crick, Bray-Curtis y 
Morisita-Horn (Raup \& Crick, 1979; Krebs, 1989). El índice de Raup-Crick es un coeficiente probabilístico que compara el número observado de taxones compartidos con la distribución de coocurrencias en 200 réplicas generadas al azar por un programa estadístico. El índice de Bray-Curtis es un coeficiente de distancia que mide las diferencias en abundancia de los taxones que componen las muestras e ignora los casos en los cuales el taxón está ausente en ambas muestras. El índice de Morisita-Horn es un coeficiente formulado para conteos de individuos, el cual mide la probabilidad que los individuos tomados de las muestras en comparación pertenezcan al mismo taxón.

Para determinar si la agregación de las matrices de datos es un proceso que mejora la aplicación de los análisis multivariados se caracterizaron las tres matrices de datos señalando el número de taxones involucrados, la cantidad de ceros y la distribución de las abundancias totales. Las distribuciones de abundancia fueron visualizadas mediante histogramas de los valores transformados a una escala logarítmica $\left(\log _{10}(\mathrm{n}+1)\right)$.

Para determinar si la estructura comunitaria estuvo relacionada al principal gradiente ambiental (aridez) del área de estudio se realizaron dos análisis con las tres combinaciones de resolución taxonómica e índice de similitud que se aproximaron con mayor fidelidad a la clasificación de puntos de muestreo basada en las características de suelo, vegetación y perturbación antrópica. Primero, los scores asignados a los puntos de muestreo en el primer eje del NMDS fueron presentados en gráficos de barras para verificar si su secuencia guarda relación con el gradiente de aridez. Segundo, las abundancias de los diez taxones con mayor abundancia total fueron correlacionadas con los scores del primer eje del NMDS para determinar la relación o afinidad de éstos con los extremos de dicho gradiente ambiental. Se utilizó el coeficiente de correlación por rangos de Spearman (rs) y se optó por un nivel de significación estadística de $\mathrm{p}<0.05$.

Todos los análisis fueron realizados con el programa estadístico PAST 3.0 (Hammer et al., 2001).

\section{Resultados.}

Los análisis multivariados realizados con las nueve combinaciones de resolución taxonómica e índice de similitud se presentan en la Figura 1. La disposición de los puntos de muestreo en el espacio bidimensional de los NMDS fue distinta para cada combinación de resolución taxonómica e índice de similitud. Los puntos del humedal de San Andrés (SA1, SA2, SA3) presentaron gran afinidad (cercanía) entre sí en la mayoría de combinaciones. Una situación similar fue observada también para los puntos del desierto sin vegetación (PF1, PF2). Estos conjuntos de puntos se sitúan en los extremos del gradiente de aridez que ha sido reconocido en el área de estudio.
Los análisis de conglomerados distinguieron dos, cinco o siete grupos de puntos de muestreo en las diferentes combinaciones de resolución taxonómica e índice de similitud. Es decir, se obtuvieron clasificaciones de puntos de muestreo insuficientes, aproximadas o excedidas con respecto a la clasificación basada en sus características ambientales. Se distinguieron dos grupos para las siguientes combinaciones: órdenes y Raup-Crick, familias y Raup-Crick, órdenes y Morisita-Horn. En los dos primeros casos, el grupo B estuvo compuesto por los puntos del humedal de San Andrés (SA1, SA2, SA3) y el grupo A compuesto por los puntos restantes. En la combinación de órdenes y Morisita-Horn, el grupo A incluyó sólo a los puntos del desierto sin vegetación (PF1, PF2), mientras que el grupo B incluyó a los puntos restantes. Se distinguieron cinco grupos para las siguientes combinaciones: órdenes y Bray-Curtis, familias y Morisita-Horn, especies y Raup-Crick, especies y Morisita-Horn. En la mayoría de casos, los grupos estuvieron compuestos por los mismos puntos, desierto sin vegetación (PF1, PF2) en el grupo A, vegetación introducida (PF3) en el grupo B, bosquecito de sofaique (SC1, SC2) en el grupo C, zona de palmeras (SC3) en el grupo D, y Humedal de San Andrés (SA1, SA2, SA3) en el grupo E. En la combinación de especies y Raup-Crick, el grupo A estuvo compuesto por los puntos del desierto sin vegetación (PF1, PF2), pero los demás grupos fueron distintos. Se distinguieron siete grupos para las siguientes combinaciones: familias y Bray-Curtis, especies y Bray-Curtis. En ambos casos, los grupos estuvieron compuestos por los mismos puntos, PF1 en el grupo A, PF2 en el grupo B, vegetación introducida (PF3) en el grupo C, bosquecito de sofaique (SC1, SC2) en el grupo D, zona de palmeras (SC3) en el grupo E, humedal poco perturbado (SA1, SA2) en el grupo $\mathrm{F}$ y humedal muy perturbado (SA3) en el grupo G.

Todos los resultados de los ANOSIM fueron estadísticamente significativos (R cercano o igual a 1, $\mathrm{p}<0.05$ ), ratificando plenamente los grupos obtenidos mediante los análisis de conglomerados. Tres combinaciones destacaron por su mayor significación estadística: órdenes y Bray-Curtis $(\mathrm{R}=1.00, \mathrm{p}=$ 0.0004), familias y Morisita-Horn $(\mathrm{R}=1.00, \mathrm{p}=$ 0.0002), especies y Morisita-Horn $(\mathrm{R}=1.00, \mathrm{p}=$ 0.0005). Estas tres combinaciones fueron también las que produjeron una clasificación de puntos de muestreo (5 grupos) más semejante a la clasificación basada en las características de suelo, vegetación y perturbación antrópica (6 grupos).

La caracterización de los datos empleados se presenta en la Figura 2. Los taxones incluidos en las matrices fueron 27 órdenes, 77 familias y 62 especies respectivamente. La cantidad de ceros ascendió a 115 en la matriz de órdenes, 450 en la matriz de familias y 421 en la matriz de especies. La distribución de 
Raup-Crick
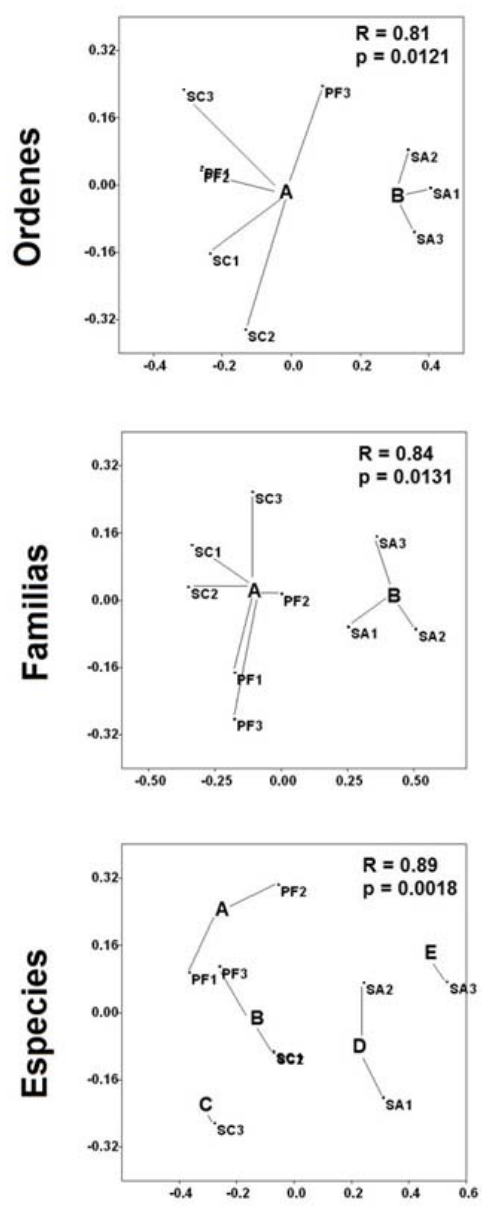

Bray-Curtis
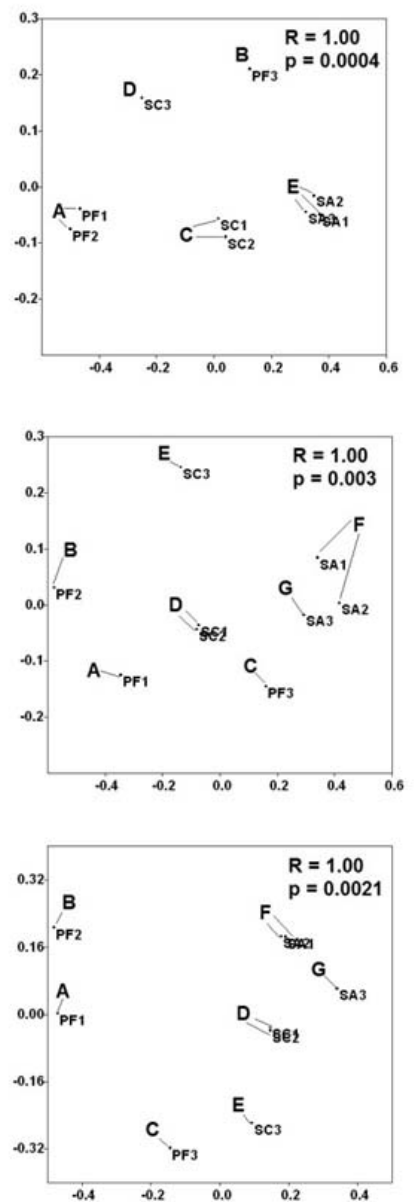

Morisita-Horn
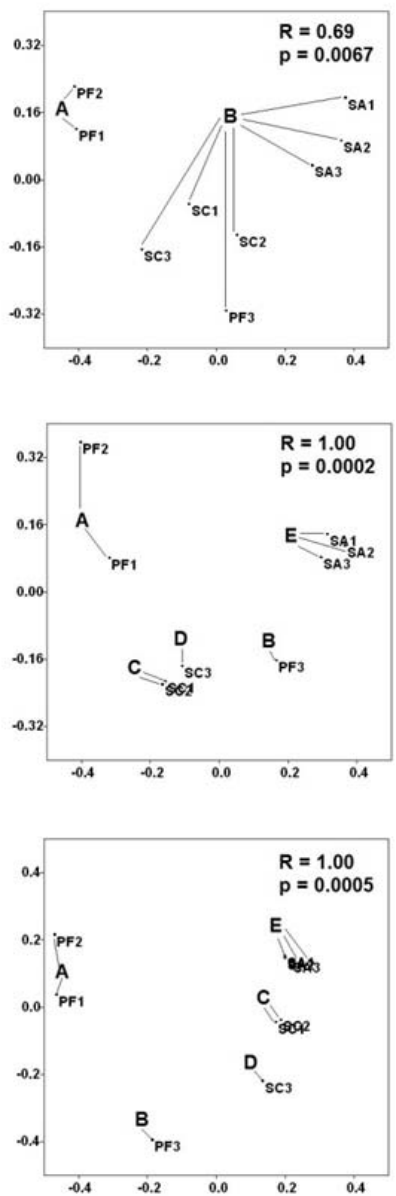

Figura 1. Análisis multivariados realizados con datos de artrópodos epígeos combinando los niveles taxonómicos de órdenes, familias y especies, con los índices de similitud de Raup-Crick, Bray-Curtis y Morisita-Horn. Letras mayúsculas designan los grupos resultantes de los análisis de conglomerados. Los resultados de los ANOSIM, se presentan a través del estadístico R y el valor de probabilidad p.

abundancias totales fue aproximadamente normal para órdenes, sesgada hacia valores menores a diez para las familias (1 en escala logarítmica), y notoriamente sesgada hacia valores menores a diez para las especies.

Las tres combinaciones de resolución taxonómica e índice de similitud con mayor significación estadística en el ANOSIM se utilizaron en análisis adicionales. Los scores de los puntos muestreo en el primer eje del NMDS para dichas combinaciones se presentan en la Figura 3. La secuencia de puntos en el NMDS de órdenes y Bray-Curtis fue PF2, PF1 y SC3 con valores negativos y SC1, SC2, PF3, SA3, SA2 y SA1 con valores positivos. La secuencia de puntos en el NMDS de familias y Morisita-Horn fue PF2, PF1, SC1, SC2 y SC3 con valores negativos y, PF3, SA3, SA1 y SA2 con valores positivos. La secuencia de puntos en el NMDS de especies y Morisita-Horn fue PF2, PF1 y PF3 con valores negativos, y SC3, SC1, SC2, SA1, SA2 y SA3 con valores positivos. Las tres secuencias de puntos de muestreo examinadas tienden a ubicar los hábitats más áridos en el extremo negativo y los hábitats menos áridos en el extremo positivo.

Las correlaciones entre la abundancia de los taxones y los scores de los puntos de muestreo en el primer eje del NMDS correspondientes a las tres combinaciones de resolución taxonómica e índice de similitud con mayor significación estadística en el ANOSIM se presentan en la Tabla 1. Para la combinación de órdenes y Bray-Curtis, ocho órdenes presentaron correlaciones positivas. Dichos órdenes fueron: Amphipoda, Diptera, Hemiptera, Actinedida, Isopoda, Araneae, Oribatida y Orthoptera. Para la combinación de familias y Morisita-Horn, cinco 
Tabla 1. Correlaciones entre los diez taxones más abundantes y los scores del primer eje del NMDS realizados con las tres combinaciones de resolución taxonómica e índice de similitud con mayor significación estadística (ANOSIM). Correlaciones efectuadas con el coeficiente de correlación de Spearman (rs). Valores en negrita corresponden a correlaciones significativas a un $\mathrm{p}$

\begin{tabular}{|c|c|c|c|}
\hline $\begin{array}{l}\text { Ordenes y } \\
\text { Bray-Curtis }\end{array}$ & $\begin{array}{c}\text { Abund. } \\
\text { total }\end{array}$ & rs & $\mathrm{p}$ \\
\hline Hymenoptera & 11841 & 0.55 & 0.13 \\
\hline Amphipoda & 3802 & 0.84 & 0.02 \\
\hline Coleoptera & 1106 & -0.23 & 0.56 \\
\hline Diptera & 784 & 0.94 & 0.00 \\
\hline Hemiptera & 630 & 0.85 & 0.01 \\
\hline Actinedida & 515 & 0.79 & 0.02 \\
\hline Isopoda & 361 & 0.75 & 0.03 \\
\hline Araneae & 331 & 0.92 & 0.00 \\
\hline Oribatida & 261 & 0.75 & 0.04 \\
\hline Orthoptera & 105 & 0.82 & 0.03 \\
\hline $\begin{array}{l}\text { Familias y } \\
\text { Morisita-Horn }\end{array}$ & $\begin{array}{l}\text { Abund. } \\
\text { total }\end{array}$ & rs & $\mathrm{p}$ \\
\hline Formicidae & 11670 & 0.42 & 0.25 \\
\hline Anobiidae & 403 & -0.34 & 0.37 \\
\hline Tenebrionidae & 323 & -0.82 & 0.01 \\
\hline Sphaeroceridae & 285 & 0.71 & 0.04 \\
\hline Aphididae & 267 & 0.04 & 0.92 \\
\hline Staphylinidae & 204 & 0.74 & 0.03 \\
\hline Lycosidae & 197 & 0.83 & 0.03 \\
\hline Cicadellidae & 106 & 0.96 & 0.00 \\
\hline Chloropidae & 96 & 0.75 & 0.03 \\
\hline Anthocoridae & 95 & -0.12 & 0.76 \\
\hline $\begin{array}{l}\text { Especies y } \\
\text { Morisita-Horn }\end{array}$ & $\begin{array}{l}\text { Abund. } \\
\text { total }\end{array}$ & rs & $\mathrm{p}$ \\
\hline Pheidole sp. & 5869 & 0.78 & 0.02 \\
\hline Wasmannia sp. & 2814 & 0.14 & 0.74 \\
\hline Dorymyrmex sp. & 2111 & -0.57 & 0.13 \\
\hline Solepnopsis sp. 02 & 408 & 0.83 & 0.01 \\
\hline Trigonogenius sp. & 391 & -0.28 & 0.47 \\
\hline Cordibates sp. & 269 & -0.73 & 0.08 \\
\hline Lycosa sp. & 197 & 0.78 & 0.04 \\
\hline Linepithema sp. & 184 & 0.52 & 0.24 \\
\hline Aleocharinae 01 & 170 & 0.84 & 0.03 \\
\hline Cardiocondyla sp. 01 & 151 & 0.68 & 0.05 \\
\hline
\end{tabular}

familias presentaron correlaciones positivas significativas y una presentó correlación negativa significativa. Dichas familias fueron: Sphaeroceridae, Staphylinidae, Lycosidae, Cicadellidae y Chloropidae con correlaciones positivas y, sólo Tenebrionidae con correlación negativa. Para la combinación de especies y Morisita-Horn, cuatro presentaron correlaciones positivas significativas. Dichas especies fueron Pheidole sp., Solenopsis sp.02, Lycosa sp., Aleocharinae 01 y Cardiocondyla sp.01.

\section{Discusión.}

La suficiencia taxonómica no provoca una significativa pérdida de información multivariada.

La interpretación conjunta de los resultados obtenidos con los NMDS, los análisis de conglomerados y los ANOSIM sugiere que las tres resoluciones taxonómicas aplicadas tuvieron el potencial de distinguir con acierto los distintos ecosistemas presentes en el área evaluada. Observaciones similares han sido reportadas para artrópodos epígeos (Pik et al., 2002; Biaggini et al., 2007; Bang \& Faeth, 2011), artrópodos epígeos y de hojarasca (Nakamura et al., 2007), Araneae, Coleoptera y Lepidoptera (Timms et al., 2013), Coleoptera y Nymphalidae (Uehara-Prado et al., 2009), Araneae (Brennan et al., 2006), Formicidae (Groc et al., 2010) y Oribatida (Caruso \& Migliorini, 2006), abarcando un amplio espectro de métodos de captura, ecosistemas y perturbaciones antrópicas.

No hay consenso acerca del grado de resolución taxonómica indicado u óptimo para aplicar la suficiencia taxonómica. En los casos citados se obtuvieron resultados positivos con géneros, subfamilias, familias y órdenes, partiendo de conjuntos de datos que abarcaron una sola familia, un solo orden o varios órdenes de artrópodos. Por otra parte, el uso de la suficiencia taxonómica no ha sido debidamente fundamentado en la teoría ecológica vigente. La redundancia funcional propone que cada flujo de energía en el ecosistema involucra una serie de especies que siendo singulares en su filogenia y autoecología, cumplen a grandes rasgos roles funcionales equivalentes (Fonseca \& Ganade, 2001). En el contexto del monitoreo biológico, posiblemente sea más inmediato detectar cambios en los flujos de energía del ecosistema que se reflejan en los taxones de alto rango, que cambios en los nichos particulares de cada especie. Al margen de la evidencia favorable, la suficiencia taxonómica no ha sido satisfactoria para diagnosticar los efectos de perturbaciones sobre comunidades de artrópodos en todos los casos. Cabe citar los siguientes: sustitución de bosques por sabanas y áreas agrícolas en varios órdenes de insectos (Basset et al., 2004), polución con metales pesados en artrópodos de suelo (Nahmani et al., 2006) y, varios tipos de perturbaciones forestales en Coleoptera (Grimbacher et al., 2008).

En cuanto a los índices de similitud, el índice de Morisita-Horn fue el más apropiado para distinguir los hábitats a partir de los datos de artrópodos epígeos, seguido por Bray-Curtis y luego Raup-Crick. Las ventajas del índice de Morisita-Horn sobre otros índices de similitud fueron resaltadas por Wolda (1981) a partir de una extensa simulación de datos. Dicho autor señaló que el índice de Morisita-Horn es casi independiente del tamaño y de la diversidad de las muestras en estudio, en tal sentido recomendó su uso en estudios de comunidades biológicas. En cuanto al 

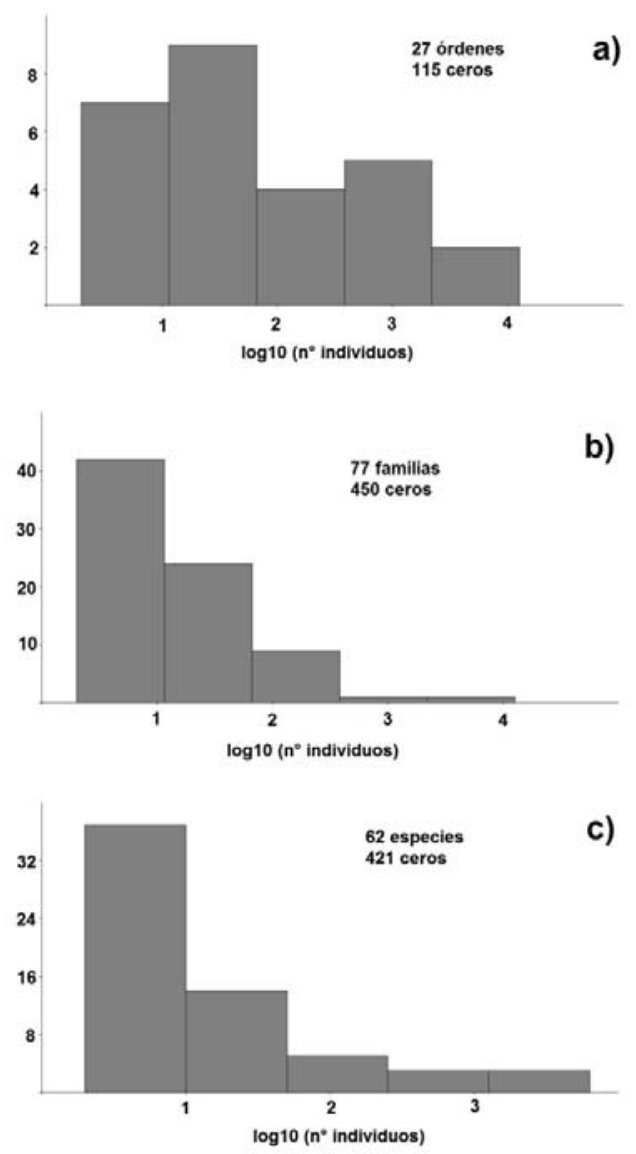

Figura 2. Ciaracterizacion de los datos utilizados en los análisis multivariados. Histogramas de abundancias totales para tres resoluciones taxonómicas, indicando número de taxones y de ceros. a) órdenes, b) familias, c) especies.

índice de Bray-Curtis, la simulación de datos efectuada por Bloom (1981) demostró su capacidad para medir el traslape de taxones entre muestras con mayor precisión que otros índices.

Agregar las matrices de datos mejora la aplicación de los análisis multivariados.

A través de la caracterización de los datos se determinó que una menor resolución taxonómica implica menor número de taxones, menor cantidad de ceros y distribuciones de abundancias más equitativas. Al aplicar un análisis multivariado dichas características se traducen respectivamente en un espacio n-dimensional más reducido (Brennan et al., 2006), una remoción del "ruido" que interfiere con otras fuentes de variación de los datos (Timms et al., 2013) y una menor cantidad de taxones poco abundantes (singletons, doubletons, etc.), los cuales no son significativos para este tipo de análisis (Basset et al., 2004). En suma, la agregación de los datos de especies en datos de menor resolución taxonómica es un proceso que favorece la aplicación de análisis multivariados, recomendable desde un punto de vista estrictamente estadístico.

Relación de la estructura comunitaria con el gradiente ambiental.

Las secuencias de puntos en el primer eje del NMDS sugieren que las abundancias de órdenes y familias estuvieron relacionadas con el gradiente ambiental de aridez, mientras que los datos de especies fueron influenciados además por la ubicación geográfica (localidades). En investigaciones recientes se ha observado que las variables ambientales (vegetación, edáficas, hídricas etc.) responsables de la distribución espacial de las comunidades de artrópodos epígeos tuvieron la misma jerarquía de importancia para diferentes resoluciones taxonómicas (Cotes et al., 2010; Schipper et al., 2010). En estos casos, el grado de heterogeneidad ambiental es tan marcado que no sólo sobrepasa la capacidad de tolerancia de las especies, sino también la tolerancia de los taxones de alto rango. Es posible que en situaciones de menor heterogeneidad ambiental, las respuestas de las comunidades sólo sean aparentes a nivel de especies. En Grimbacher et al. (2008), los datos de Coleoptera analizados a la resolución de familias fueron útiles para diagnosticar la sustitución de bosques por pastizales, mientras que los datos de especies permitieron diagnosticar esta perturbación y otras más sutiles, incluyendo fragmentación y un gradiente climático latitudinal.

Una revisión de los órdenes, familias y especies que presentaron correlaciones significativas, revela que éstos abarcaron un amplio espectro filogenético y funcional. Es decir, no fue un único taxón sino combinaciones de distintos taxones, los que pueden reflejar los patrones del conjunto de artrópodos epígeos y en tal sentido actuar como potenciales indicadores. Este enfoque multitaxa en la selección de taxones indicadores ha encontrado evidencias favorables en bosques tropicales africanos (Basset et. al., 2004), bosques montanos africanos (Lawes et al., 2005) y en el Bosque Atlántico de Brasil (UeharaPrado et al., 2009). Aunque la Tabla 1 presenta conjuntos de artrópodos que podrían servir como indicadores en ecosistemas del desierto peruano, es preciso tomarlos con precaución hasta que se lleven a cabo estudios similares al presente en otras localidades.

La mayoría de taxones presentaron correlaciones positivas con el 1er eje del NMDS, es decir estuvieron asociados a los ecosistemas con un suministro regular de agua, la vegetación introducida (PF3) y el Humedal de San Andrés (SA1, SA2, SA3). Es probable que este tipo de ecosistemas sean preferidos debido a su mayor productividad y sus temperaturas moderadas, como ha sido observado en paisajes desérticos de Norteamérica (McIntyre et al., 2001; Shochat et al., 2004). En 

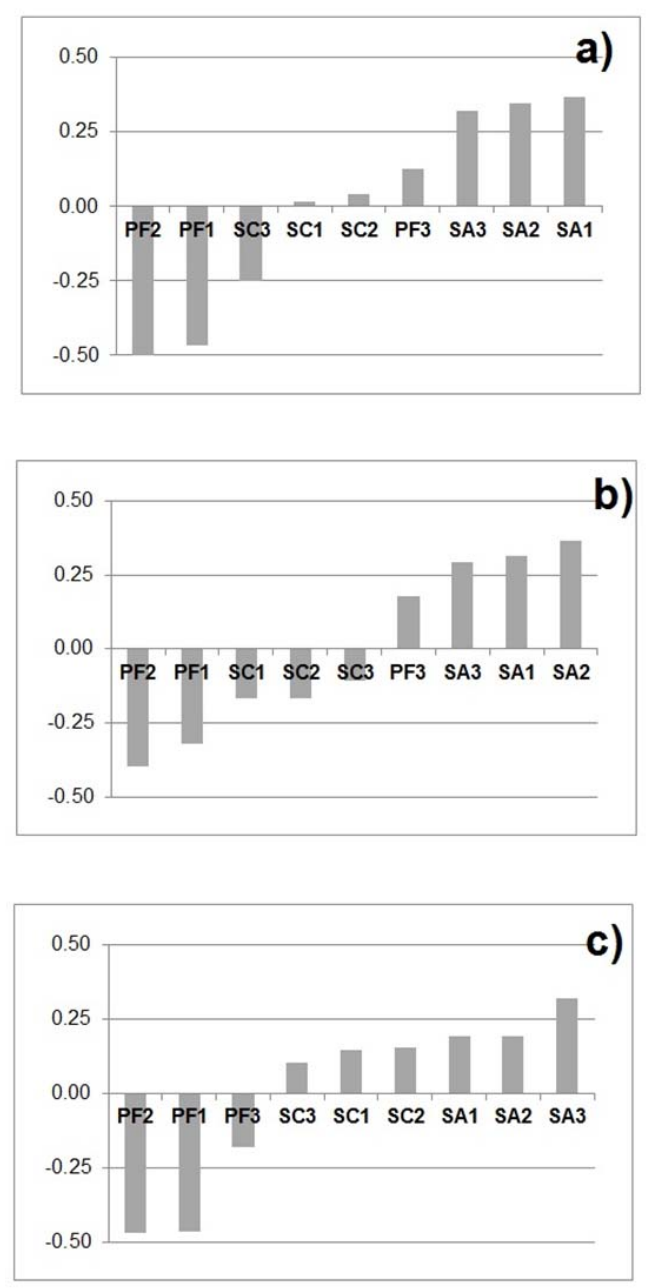

Figura 3. Secuencia de puntos de muestreo en el primr eje de los NMDS realizados con tres combinaciones de resolución taxonómica e índice de similitud: a) órdenes y Bray-Curtis, b) familias y Morisita-Horn, c) especies y Morisita-Horn.

contraste, sólo algunos taxones cuentan con adaptaciones morfológicas, fisiológicas y etológicas para subsistir e incluso preferir ecosistemas áridos, es el caso de la familia Tenebrionidae (Crawford, 1981). Recomendaciones para futuros estudios.

A partir de los resultados obtenidos se recomienda que los análisis de datos comunitarios de artrópodos terrestres sean ante todo flexibles. El presente trabajo ha demostrado que la distinción de ecosistemas y la descripción de gradientes ambientales pueden obtenerse a partir distintas combinaciones de resolución taxonómica e índice de similitud. Es conveniente pasar por esta etapa exploratoria en el análisis de datos, y escoger la combinación que represente el mejor balance entre esfuerzo e información. Asimismo, una caracterización de datos similar a la presentada aquí, sería útil para observar los cambios provocados por el proceso de agregación.

Finalmente, señalar que los resultados del presente estudio estuvieron limitados por cuatro factores: el breve tiempo operativo de las trampas de caída, el limitado número de puntos de muestreo por ecosistema, la carencia de datos cuantitativos para las variables ambientales y la limitada representación de los ecosistemas presentes en el paisaje del desierto costero. Por tanto, es oportuno recomendar un mayor número de réplicas por ecosistema y una mayor cobertura de ecosistemas para futuros estudios que tengan como objetivo poner a prueba la suficiencia taxonómica como metodología para el monitoreo de comunidades de artrópodos terrestres.

\section{Conclusiones.}

- La estructura comunitaria de artrópodos epígeos analizada a tres niveles de resolución taxonómica (órdenes, familias, especies), tuvo la capacidad de distinguir los ecosistemas incluidos en el área de estudio.

- Reducir la resolución taxonómica implica agregar las matrices de datos, dándoles características que favorece la aplicación de los análisis multivariados.

- La estructura comunitaria de artrópodos epígeos a distintas resoluciones taxonómicas estuvo relacionada al gradiente ambiental que subyace y distingue a los ecosistemas evaluados.

\section{Agradecimientos.}

El autor agradece a ERM Perú S. A., Pluspetrol Perú Corporation S. A. y Reserva Nacional de Paracas por las facilidades logísticas y financieras proporcionadas dentro del Proyecto Monitoreo Biótico y Abiótico para el componente Marítimo de la Planta de Fraccionamiento de LGN, Playa Lobería, Pisco. A Gabriella González Blacker por los valiosos comentarios hechos al manuscrito. A Mariajosé Deza Bouroncle por la determinación de las familias y especies de Araneae.

\section{Literatura citada.}

Aguilera M. A. \& Casanueva M. E. 2005. Arañas chilenas: Estado actual del conocimiento y clave para las familias de Araneomorphae. Gayana, 69(2): 201-224.

Arnett R. H. 1985. American Insects. A handbook of the insects of America north of Mexico. Van Nostrand Reinhold Company. New York (USA). 850 p.

Ausden M. 1996. Invertebrates. In: Ecological Census Techniques a handbook. Edited by William Sutherland. Cambridge University Press. 336 p.

Bang C. \& Faeth S. H. 2011. Variation in arthropod communities in response to urbanization: Seven years of arthropod monitoring in a desert city. Landscape and Urban Planning, 103: 383-399. 
Barrientos J. A. (ed.) 1988. Bases para un curso práctico de entomología. Asociación española de Entomología. Barcelona (España). 754 p.

Basset Y., Mavoungou J. F., Mikissa J. B., Missa O., Miller S. E., Kitching R. L. \& Alonso A. 2004. Discriminatory power of different arthropod data sets for the biological monitoring of anthropogenic disturbance in tropical forests. Biodiversity and Conservation, 13: 709-732.

Biaggini M., Consorti R., Dapporto L., Dellacasa M., Paggetti E. \& Corti C. 2007. The taxonomic level order as a possible tool for rapid assessment of arthropod diversity in agricultural landscapes. Agriculture, Ecosystems and Environment, 122: 183-191.

Bloom S. A. 1981. Similarity indices in community studies: potential pitfalls. Marine Ecology Progress Series, 5: 125-128.

Brennan K. E. C., Ashby L., Majer J. D., Moir M. L. and Koch J. M. 2006. Simplifying assessment of forest management practices for invertebrates: How effective are higher taxon and habitat surrogates for spiders following prescribed burning? Forest Ecology and Management, 231(1-3): 138-154.

Cardoso P., Erwin T. L., Borges P. A.V. \& New T. R. 2011. The seven impediments in invertebrate conservation and how to overcome them. Biological Conservation 144, 2647-2655.

Caruso T. \& Migliorini M. 2006. Micro-arthropod communities under human disturbance: is taxonomic aggregation a valuable tool for detecting multivariate change? Evidence from Mediterranean soil oribatid coenoses. Acta Oecologica, 30: 46-53.

Cotes B., Campos M., Pascual F., García P. A. \& Ruano F. 2010. Comparing taxonomic levels of epigeal insects under different farming systems in Andalusian olive agroecosystems. Applied Soil Ecology, 44: 228-236.

Crawford C. S. 1981. Biology of desert invertebrates. Springer-Verlag Berlin Heildelberg. New York (USA). $291 \mathrm{p}$.

[CSIRO] (ed.) 1991. The Insects of Australia. Second Edition. Volume 1 and 2. Commonwealth Scientific and Industrial Research Organization (CSIRO). The Division of Entomology. Melbourne University Press. Canberra (Aresolustralia). 1137 p.

Ellis D. 1985. Taxonomic sufficiency in pollution assessment. Marine Pollution Bulletin, 16: 459.

Fonseca C. R. \& Ganade G. 2001. Species functional redundancy, random extinctions and the stability of ecosystems. Journal of Ecology, 89: 118-125.

Grimbacher P. S., Catterall C. P. \& Kitching R. L. 2008. Detecting the effects of environmental change above the species level with beetles in a fragmented tropical rainforest landscape. Ecological Entomology, 33: 66-79.

Groc S., Delabie J.H.C., Longino J.T., Orivel J., Majer J.D.,Vasconcelos H.L. \& Dejean A. 2010. A new method based on taxonomic sufficiency to simplify studies on Neotropical ant assemblages. Biological Conservation, 143: 2832-2839.

Hammer Ø., Harper D.A.T. \& Ryan P.D. 2001. PAST: Paleontological statistics software package for education and data analysis. Palaeontologia Electronica 4(1): 9pp. http://palaeo-electronica.org/2001_1/past/issue1_01.htm

Jones F. C. 2008. Taxonomic sufficiency: The influence of taxonomic resolution on freshwater bioassessments using benthic macroinvertebrates. Environmental Revue, 16: 45-69.

Krebs C. J. 1989. Ecological Methodology. University of British Columbia. Harper Collins Publishers. New York (USA). 654 p.

Lawes M. J., Kotze D. J., Bourquin S. L. \& Morris C. 2005. Epigaeic invertebrates as potential ecological indicators of Afromontane Forest condition in South Africa. Biotropica, 37(1): 109-118.

Legendre P. \& Gallagher E. D. 2001. Ecologically meaningful transformations for ordination of species data. Oecologia, 129: 271-280.

Legendre P. \& Legendre L. 1998. Numerical Ecology. Second English Edition. Developments in Environmental Modelling 20. Elsevier Science B. V. Amsterdam. 853 p.

Major R. E., Smith D., Cassis G., Gray M. \& Colgan D. J. 1999. Are roadside strips important reservoirs of invertebrate diversity? A comparison of the ant and beetle faunas of roadside strips and large remnant woodlands. Australian Journal of Zoology, 47: 611-624.

Márquez-Luna J. 2005. Técnicas de colecta y preservación de insectos. Boletín Sociedad Entomológica Aragonesa, 37: 385-408.

Martínez C. 2005. Introducción a los escarabajos Carabidae (Coleoptera) de Colombia. Instituto de Investigación de Recursos Biológicos Alexander von Humboldt. Bogotá D. C., Colombia. 546 p.

Mazur S. 2001. Review of the Histeridae (Coleoptera) of Mexico. Dugesiana, 8(2): 17-66.

McAlpine J. F., Peterson B. V., Shewell G. E., Teskey H. J., Vockeroth J. R. \& Wood D. M. (eds.) 1981. Manual of Nearctic Diptera. Vol. 1. Research Branch Agriculture Canada. Monograph ${ }^{\circ} 27$. Ontario (Canada).

McIntyre N. E., Rango J., Fagan W. F. \& Faeth S. H. 2001. Ground arthropod community structure in a heterogeneous urban environment. Landscape and Urban Planning, 52: 257-274.

May R. M. 1990. How many species? Philosophical Transactions of the Royal Society of London. Series B, 330: 293-304.

Nahmani J., Lavelle P. \& Rossi J. -P. 2006. Does changing the taxonomical resolution alter the value of soil macroinvertebrates as bioindicators of metal pollution? Soil Biology \& Biochemistry, 38: 385-396.

Nakamura A., Catterall C. P., House A. P. N., Kitching R. L. \& Burwell C. J. 2007. The use of ants and other soil and litter arthropods as bio-indicators of the impacts of rainforest clearing and subsequent land use. Journal of Insect Conservation, 11(2): 177-186.

Navarrete-Heredia J. L., Newton A. F., Thayer M. K., Ashe J. S. \& Chandler D. S. 2002. Guía ilustrada para los géneros de Staphylinidae (Coleoptera) de México. Universidad de Guadalajara. Comisión Nacional para el conocimiento y uso de la biodiversidad (CONABIO). México.

New T. R. 1996. Taxonomic focus and quality control in insect surveys for biodiversity conservation. Australian Journal of Entomology, 35: 97-106.

Oliver I. \& Beattie J. 1996. Invertebrate morphospecies as surrogates for species: A case study. Conservation Biology, 10(1): 99-109.

Palacio E. E. \& Fernández F. 2003. Clave para las subfamilias y géneros. Capítulo 15 en: Fernández F. 
(ed.). Introducción a las Hormigas de la región Neotropical. Instituto de Investigación de Recursos Biológicos Alexander von Humboldt. Bogotá (Colombia). $398 \mathrm{p}$.

Pik A. J., Dangerfield J. M., Bramble R. A., Angus C. \& Nipperess D. A. 2002. The use of invertebrates to detect small-scale habitat heterogeneity and its application to restoration practices. Environmental Monitoring and Assessment, 75: 179-199.

Pik A. J., Oliver I. \& Beattie A. J. 1999. Taxonomic sufficiency in ecological studies of terrestrial invertebrates. Australian Journal of Ecology, 24: 555562.

Raup D. \& Crick R. E. 1979. Measurement of faunal similarity in paleontology. Journal of Paleontology 53:1213-1227.

Schipper A. M., Lotterman K., Geertsma M., Leuven R. S. E. W. \& Hendriks A. J. 2010. Using datasets of different taxonomic detail to assess the influence of floodplain characteristics on terrestrial arthropod assemblages. Biodiversity and Conservation, 19: 2087-2110.

Shochat E., Stefano W. L., Whitehouse M. E. A. \& Faeth S. H. 2004. Urbanization and spider diversity: influences of human modification of habitat structure and productivity. Ecological Applications, 14(1): 268-280.

Slotow R. \& Hamer M. 2000. Biodiversity research in South Africa: comments on current trends and methods. South African Journal of Science, 96: 222-224.
Terlizzi A., Bevilacqua S., Fraschetti S. \& Boero F. 2003. Taxonomic sufficiency and the increasing insufficiency of taxonomic expertise. Marine Pollution Bulletin, 46: 556-561.

Timms L. L., Bowden J. J., Summerville K. S. \& Buddle C. M. 2013. Does species-level resolution matter? Taxonomic sufficiency in terrestrial arthropod biodiversity studies. Insect Conservation and Diversity, 6: 453-462.

Uehara-Prado M., de Oliveira Fernandes J., de Moura Bello A., Machado G., Santos A. J., Zagury Vaz de Mello F. \& Lucci Freitas A. V. 2009. Selecting terrestrial arthropods as indicators of small-scale disturbance: A first approach in the Brazilian Atlantic Forest. Biological Conservation, 142: 1220-1228.

Vitousek P. M., Mooney H. A., Lubchenco J. \& Melillo J. M. 1997. Human domination of Earth's ecosystems. Science, 277: 494-499.

Ward D. F. \& Larivière M.-C. 2004. Terrestrial invertebrate surveys and rapid biodiversity assessment in New Zealand: lessons from Australia. New Zealand Journal of Ecology, 28(1): 151-159.

Warwick R. M. \& Clarke K. R. 1993. Comparing the severity of disturbance: a metaanalysis of marine macrobenthic community data. Marine Ecology Progress Series, 92: 221-231.

Wolda H. 1981. Similarity indices, sample size and diversity. Oecologia (Berlin), 50: 296-302.

\footnotetext{
${ }^{1}$ Dirección electrónica: alfre2cotobius@yahoo.com. Museo de Entomología “Klaus Raven Büller” - Universidad Nacional Agraria La Molina. Av. La Molina S/N, La Molina, Lima (Perú), Apartado Postal 12-056.
} 